\begin{abstract}
تأثير تغليف بيض المائدة بالزيوت الطبيعية وظروف الخزن في صلاحيته للاستهلاك
شهززاد محمد جعفر ألثديدي

مركز إحياء التزراث العلمي العربي / جامعة بغداد

الخلاصة

إن هدف البحث تغليف بيض المائدة ببعض الأنواع من الزيوت النباتية الطبيعية و اثر ذلك في صلاحيته للاستهلاك الته

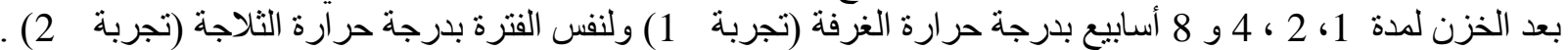
استخدم بيض مائدة طاز ج من دجاج بياض تجاري ل

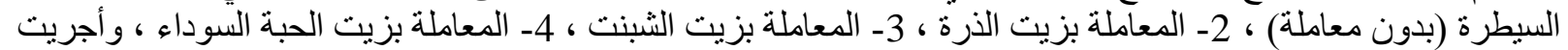

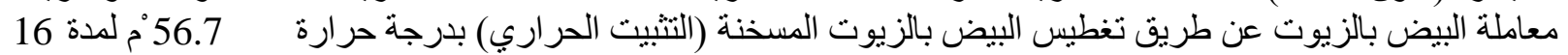

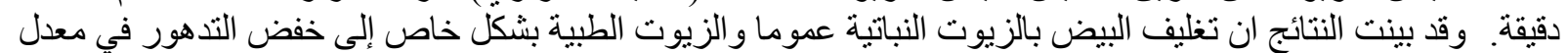

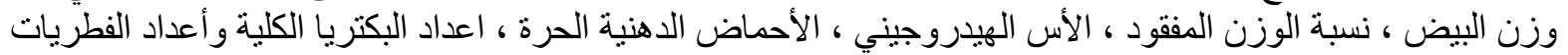

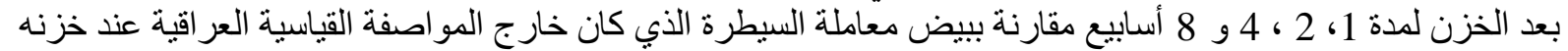

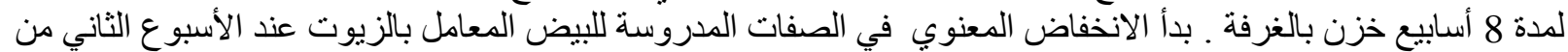

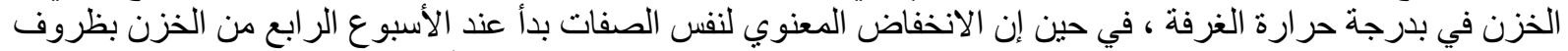

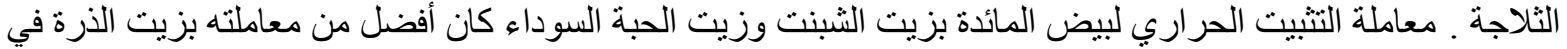

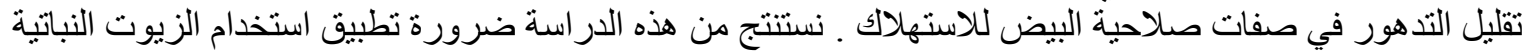

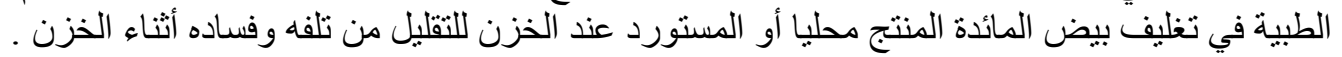

\title{
EFFECT OF COATING TABLE EGGS WITH NATURAL OILS AND STORAGE CONDITIONS ON ITS SHELF LIFE
}

\author{
Shahrazad M. J. Al-Shadeedi \\ Arab Scientific Heritage Revival Center / University of Baghdad
}

Accepted -October -2010

\section{Summary}

The object of this study was to evaluate coating table eggs with different medicinal oils and their effects on shelf life properties of fresh eggs. After storage for 1, 2, 4 and 8 weeks at room temperature (experiment 1) and refrigerator temperature (experiment 2). Fresh table eggs were collected from a flock of Isa brown laying hens, the eggs were distributed into four groups as follow :-1-Control group 2-Corn oil group 3-Dill oil group 4-Black seed oil group, the eggs were coated by immersioned in oil at $56.7^{\circ} \mathrm{C}$ for $16 \mathrm{~min}$ (Oil thermostabilization). The results revealed that coating table eggs with oils in general and specially medicinal oils reduced the mean decrease in egg weight, egg weight loss , PH, free fatty acids, total bacterial count and fungi count after storage for 1, 2, 4 and 8 weeks compared with the control . Control group eggs expired after 8 weeks of storage at room conditions according to Iraq standard specification for eggs.

E.mail: dr_shahrazad08@yahoo.com المر اسلة : شهرز اد محمد ألثديدي 
The significant reduction in shelf life properties of the eggs treated with natural oils started at the second week under room temperature storage, and fourth week under refrigerator temperature. Dill oil or black seed oil thermo stabilization for table eggs were better than corn oil in reduced the reduction of shelf life after storage at room or refrigerator temperatures. Conclusion, coating locally produced or imported table eggs with medicinal oils to prevention the eggs from deterioration and spoilage during storage.

Key words: medicinal oils, table eggs, storage, shelf life.

المقدمة

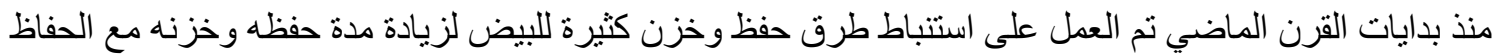

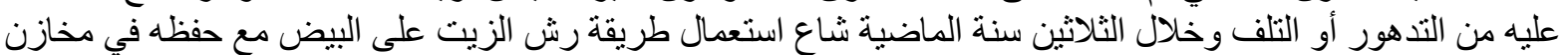

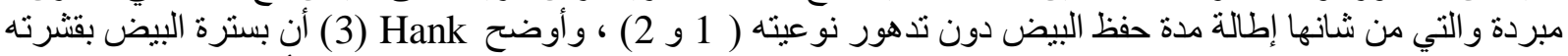

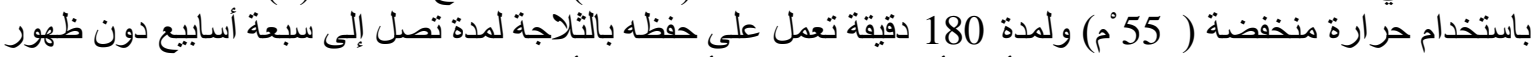

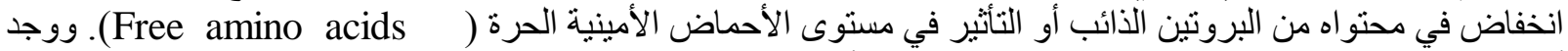

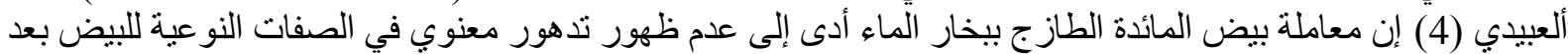

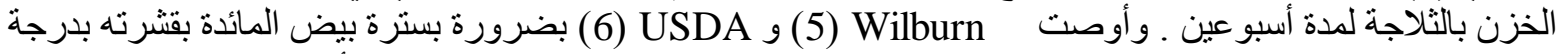

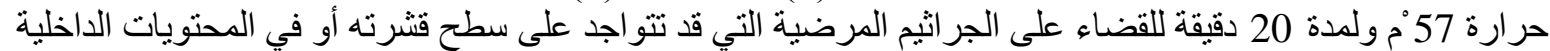

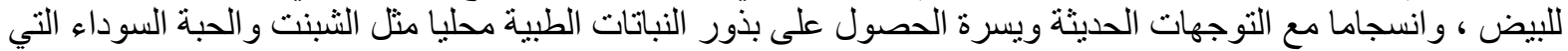

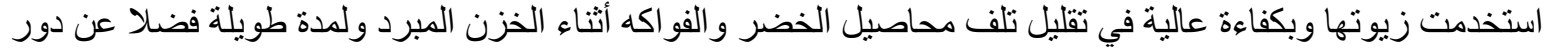

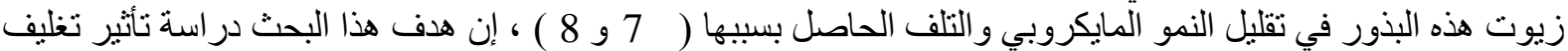

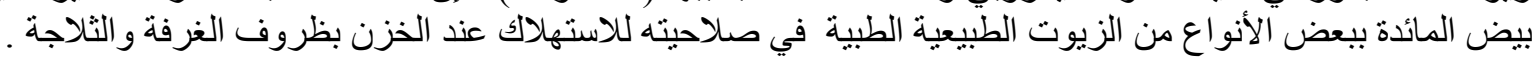

\section{المواد وطرائق العمل}

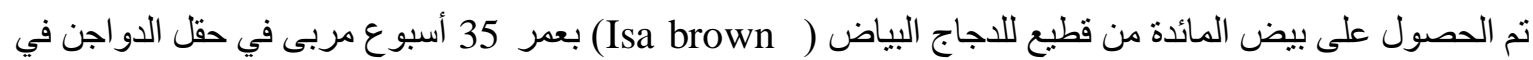

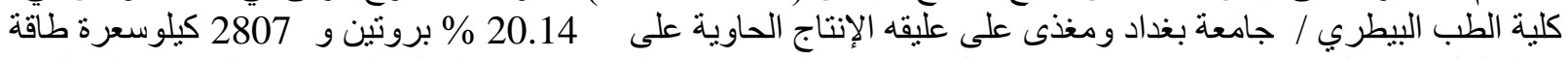

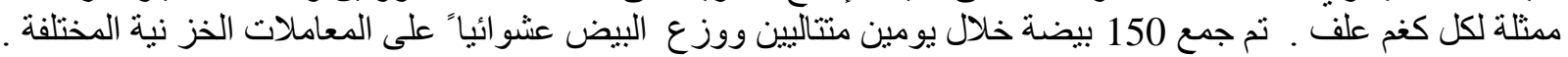
تم تقسيم المعاملات الخز نية إلى قسمين رئيسيين : تصنيف

أولاً : الخزن بدرجة حرارة الغرفة حيث تم وضع 150 بيضة بدرجة حرارة نراوحت بين 15 إلى 22 م o لمدة 1، 2 ، 4 و8 أسابيع.

ثانياً : الخزن بدرجة حرارة الثلاجة حيث تم وضع 150 بيضة بدرجة حر ارة تر اوحت بين 4 - 5 م ملمدة 1، 2 ، 4 و 8

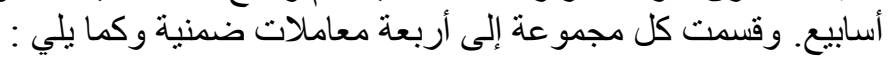

1 - معاملة المقارنة (Control) و هو البيض غير المعامل (75 بيضة ) ماركة ) .

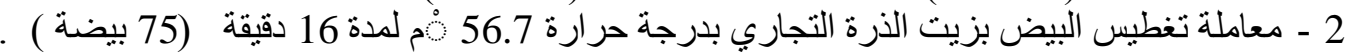

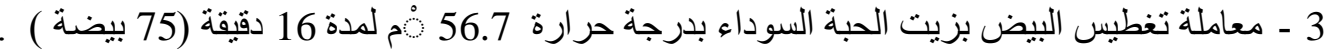

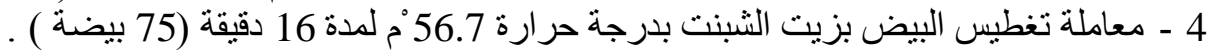

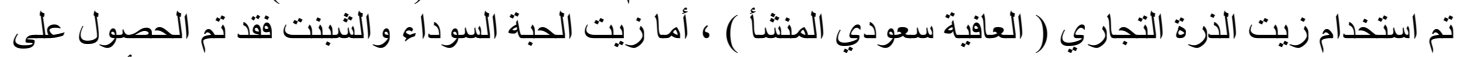

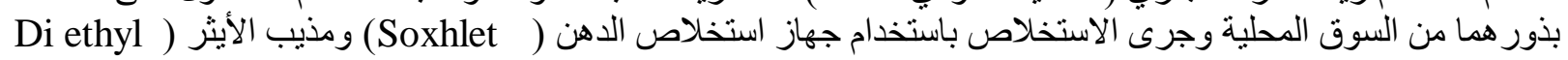
ب ether

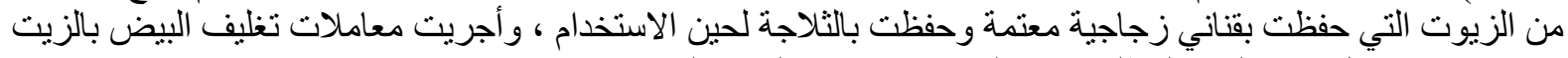

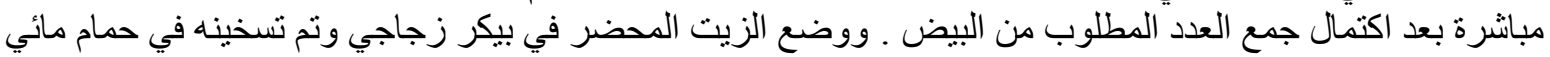

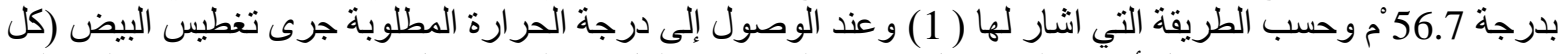

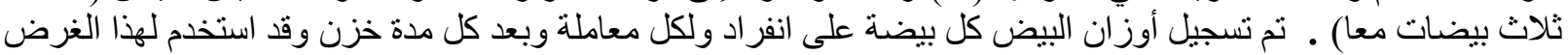

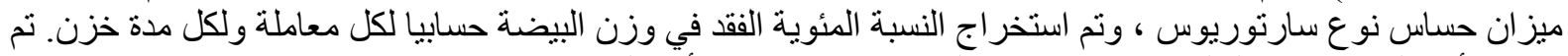

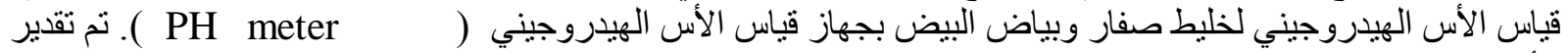

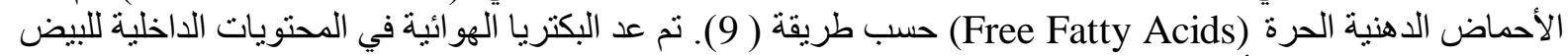

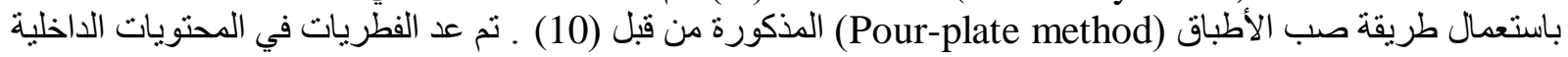




\section{المجلة الطبية البيطرية العراقية 34 ( 2 ): 131 - 141 ، ( 2010 ).}

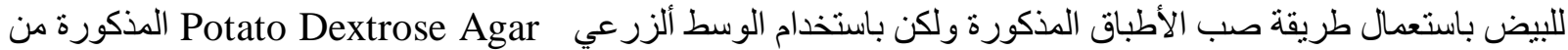

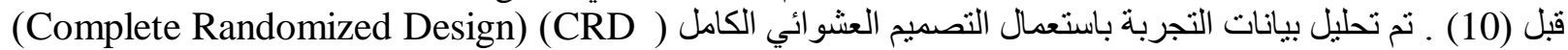

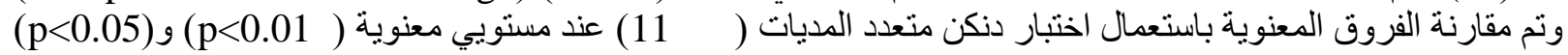

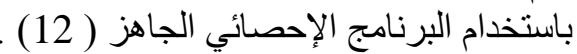

\section{النتائج}

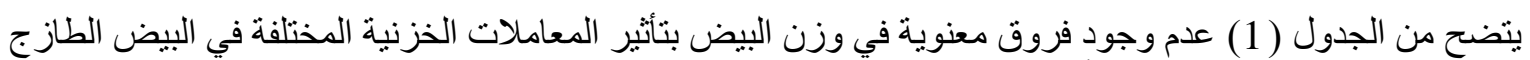

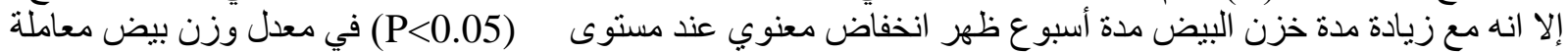

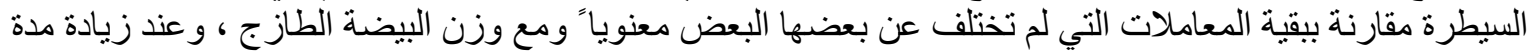

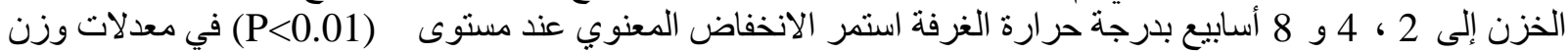

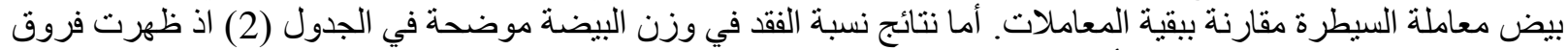

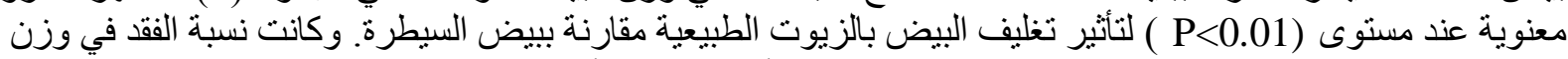

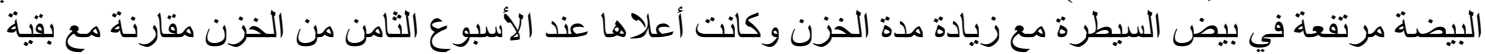

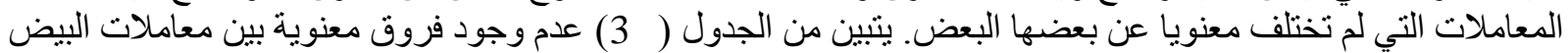

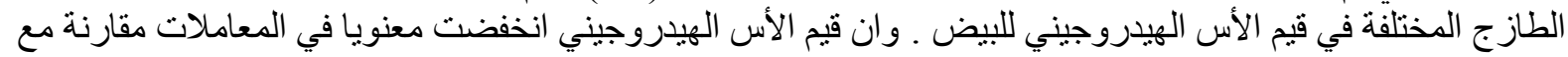

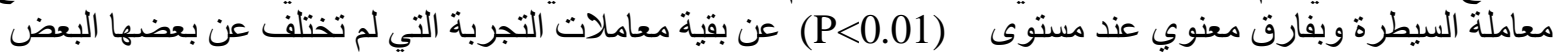

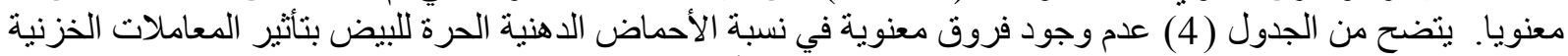

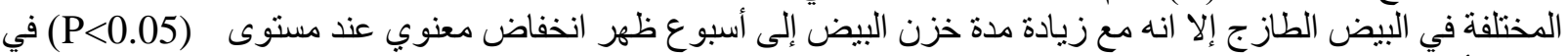

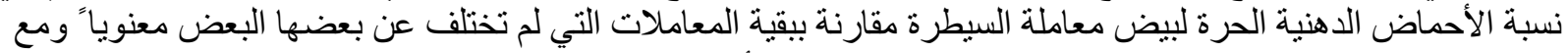

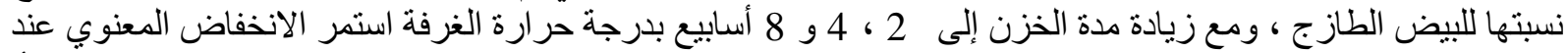

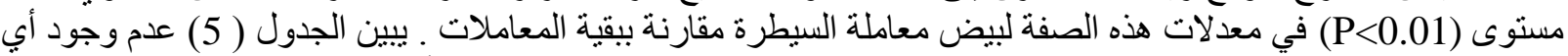

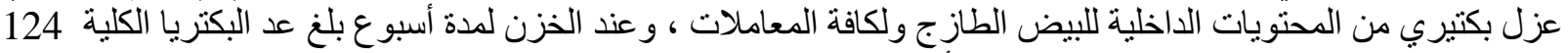

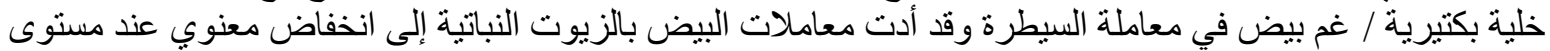

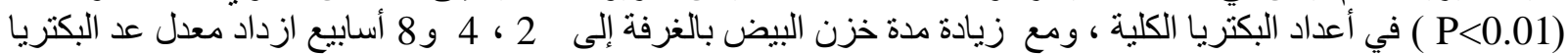

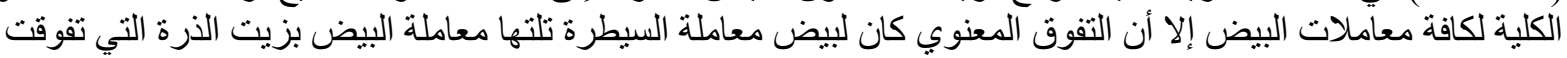

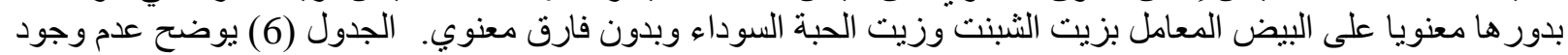

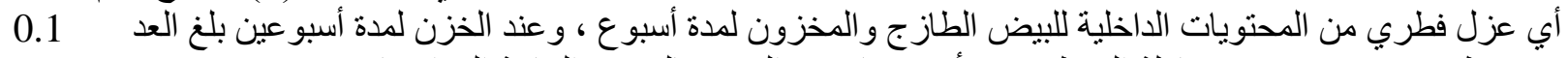

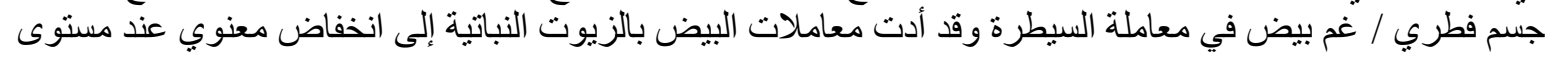

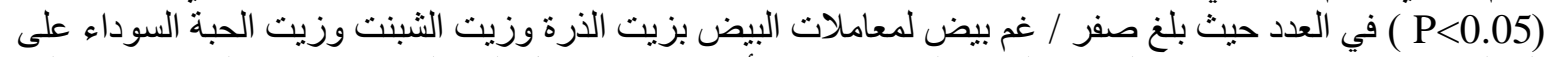

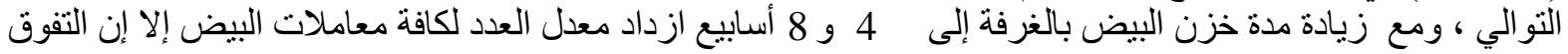

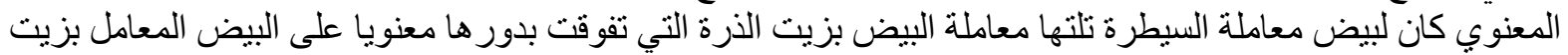

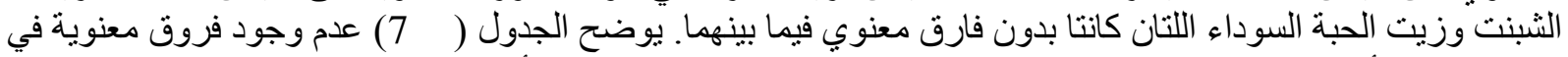

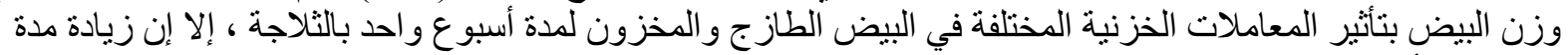

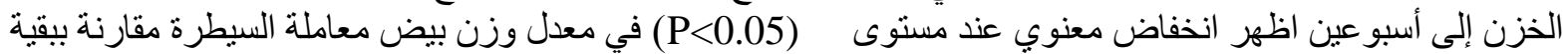

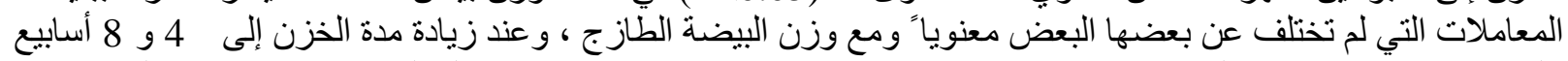

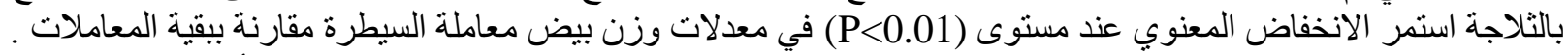

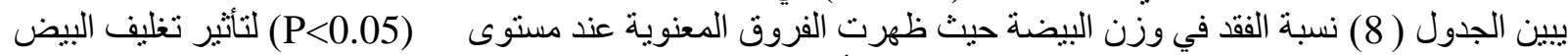

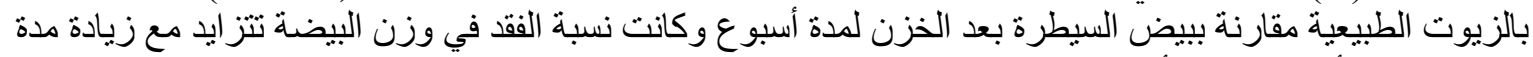

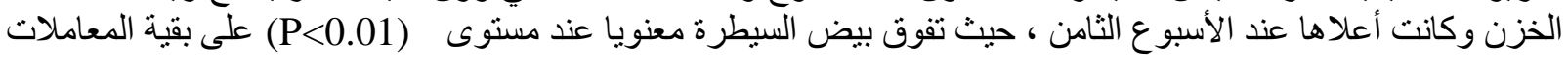

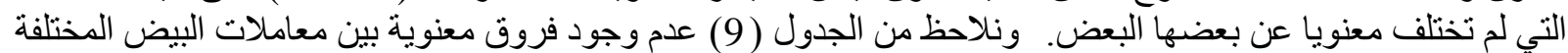

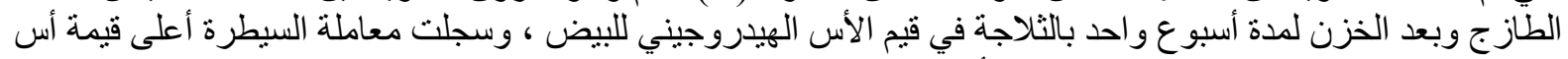

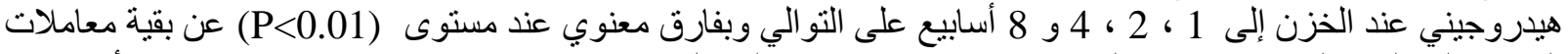

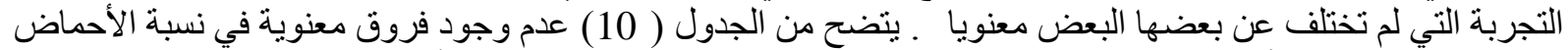

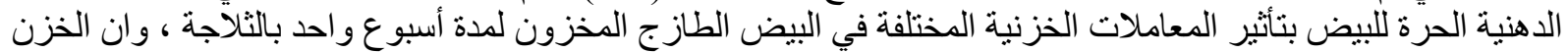

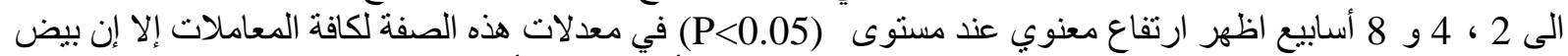

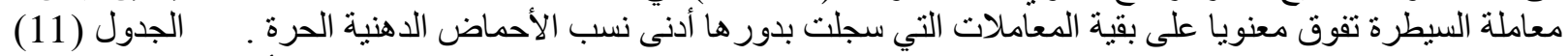

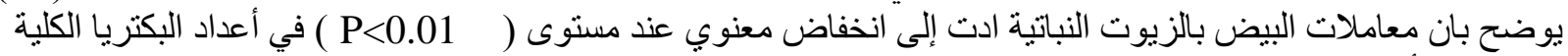

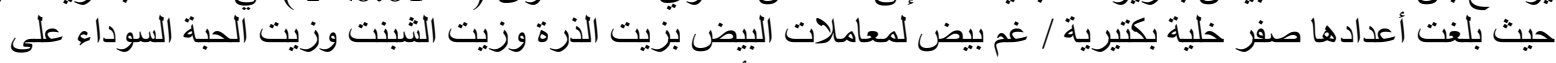

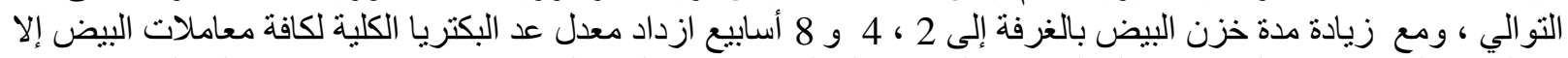

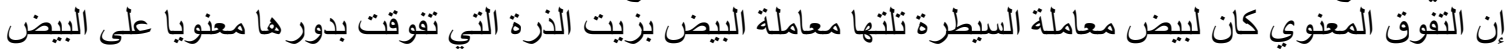




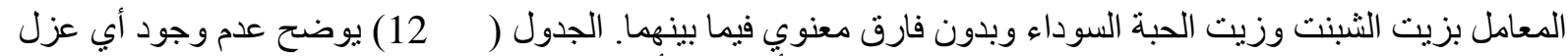

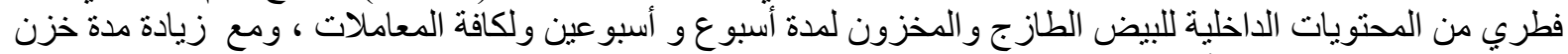

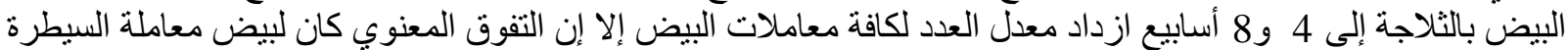

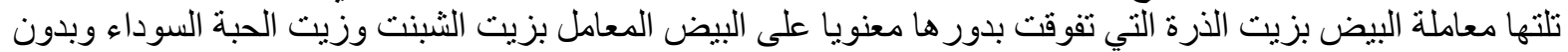
فارق معنوي فيما بينهما.

\section{المناقثة}

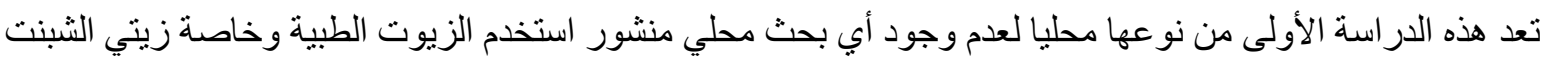

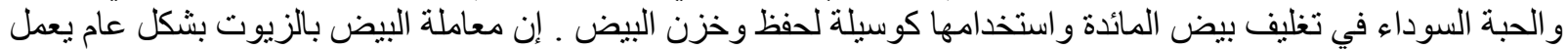

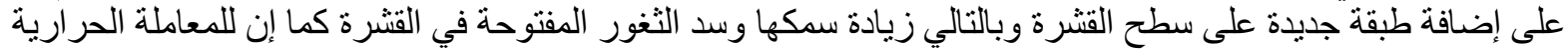

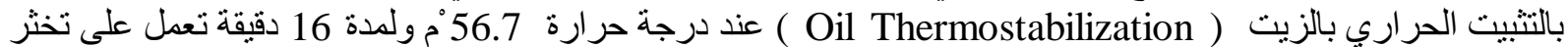

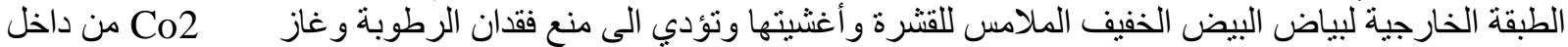
البيضة عبر الثغور إلى اقل حد ممكن أثناء الخزن وبالتالي إطالة العمر ألخزني للبيض (

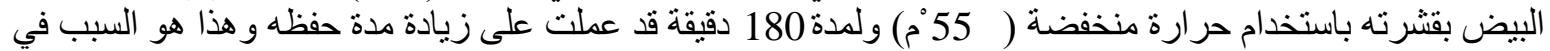

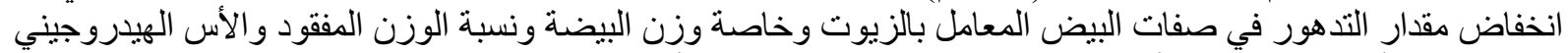

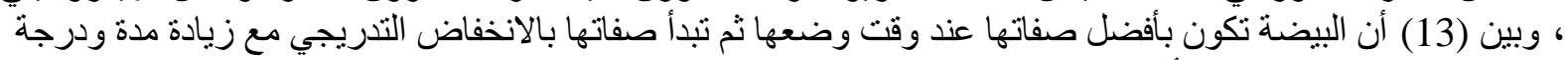

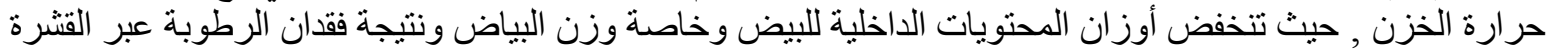

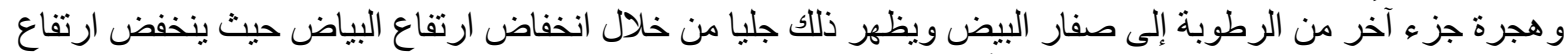
البياض السميك بين 0.05 - 0.102 ملم / أسبوع من الخزن ( 14) و الذي يؤدي إلى انخفاض وزن البيضة والئي العديد من

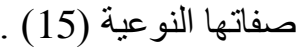

جدول ( 1 ) تأثير المعاملات المختلفة ومدة خزن البيض في الغرفة في معدل وزن البيضة (غم).

\begin{tabular}{|c|c|c|c|c|c|c|}
\hline تأثنير مدة & \multicolumn{4}{|c|}{ مدة الخزن (أسبوع) } & & \multirow{2}{*}{ المعاملات } \\
\hline الخزن & 8 & 4 & 2 & 1 & طازج & \\
\hline ** & $\begin{array}{c}46.16 \pm 1.35 \\
\text { b } E\end{array}$ & $\begin{array}{c}50.52 \\
\text { b }\end{array}$ & $\begin{array}{c}54.68 \pm 1.11 \\
\text { b } \\
\text { C }\end{array}$ & $\begin{array}{c}59.08 \\
\pm 1.20 \mathrm{~b} \\
\\
\text { B }\end{array}$ & $\begin{array}{c}61.21 \\
\pm 1.23 \mathrm{~A}\end{array}$ & سيطرة \\
\hline$* *$ & $\begin{array}{c}50.94 \pm 1.24 \\
\text { a } \quad \text { D }\end{array}$ & $\begin{array}{c}53.78 \pm 1.26 \\
\text { a } \quad \mathrm{C}\end{array}$ & $\begin{array}{c}56.91 \pm 1.19 \\
\text { a } \\
\text { B }\end{array}$ & $\begin{array}{c}60.21 \\
\pm 1.19 \mathrm{a} \\
\mathrm{A}\end{array}$ & $\begin{array}{c}61.39 \\
\pm 1.19 \mathrm{~A}\end{array}$ & زيت الذرة \\
\hline$* *$ & $\begin{array}{c}50.97 \pm 1.27 \\
\text { a } \quad \text { D }\end{array}$ & $\begin{array}{c}53.69 \pm 1.22 \\
\text { a } \quad \mathrm{C}\end{array}$ & $\begin{array}{c}56.88 \pm 1.24 \\
\text { a } \\
\text { B }\end{array}$ & $\begin{array}{c}60.28 \\
\pm 1.27 \text { a } \\
\text { A }\end{array}$ & $\begin{array}{c}61.47 \\
\pm 1.22 \mathrm{~A}\end{array}$ & زيت الشبنت \\
\hline$* *$ & $\begin{array}{c}51.01 \pm 1.18 \\
\text { a } D\end{array}$ & $\begin{array}{c}53.73 \pm 1.25 \\
\text { a } \quad \mathrm{C}\end{array}$ & $\begin{array}{c}56.86 \pm 1.18 \\
\text { a } \\
\text { B }\end{array}$ & $\begin{array}{c}60.27 \\
\pm 1.33 \mathrm{a} \\
\mathrm{A}\end{array}$ & $\begin{array}{r}61.46 \\
\pm 1.04 \mathrm{~A}\end{array}$ & زيت الحبة السوداء \\
\hline & $* *$ & $* *$ & $* *$ & * & N.S. & تأثئير المعاملات \\
\hline
\end{tabular}

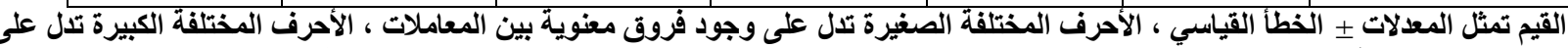

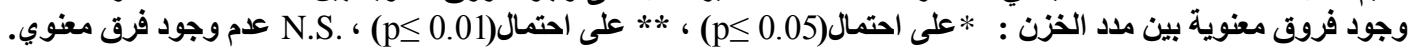


المجلة الطبية البيطرية العراقية 34 ( 2 ): 131 - 141 ، ( 2010 ).

جدول ( 2 ) تأثير المعاملات المختلفة ومدة خزن البيض في الغرفة في نسبة الفقد في وزن البيضة (\%) .

\begin{tabular}{|c|c|c|c|c|c|c|}
\hline تأثير مدة & \multicolumn{4}{|c|}{ مدة الخزن (اسبوع) } & & \multirow{2}{*}{ المعاملات } \\
\hline الخزن & 8- 1 أسابيع & 8 & 4 & 2 & 1 & \\
\hline$* *$ & $\begin{array}{c}27.17 \pm 0.48 \\
a\end{array}$ & $\begin{array}{c}8.63 \pm 0.42 \\
\text { a } \quad A\end{array}$ & $\begin{array}{cc}7.61 & \pm 0.47 \\
\text { a } & A\end{array}$ & $\begin{array}{c}7.45 \pm 0.52 \\
\text { a } \quad \mathrm{A}\end{array}$ & $\begin{array}{c}3.48 \\
\pm 0.59 \text { a } \\
\text { B }\end{array}$ & سبطرة \\
\hline$* *$ & $\begin{array}{c}18.15 \\
b\end{array}$ & $\begin{array}{c}5.28 \\
\text { b }\end{array} \quad$ A & $\begin{array}{c}5.50 \pm 0.52 \\
\text { b } A\end{array}$ & $\begin{array}{c}5.53 \pm 0.54 \\
\text { b } \quad \text { A }\end{array}$ & $\begin{array}{c}1.87 \pm 0.56 \\
\text { b } \\
\text { B }\end{array}$ & زيت الذرة \\
\hline$* *$ & $\begin{array}{c}18.25 \\
b\end{array}$ & $\begin{array}{c}5.07 \\
\text { b } \\
\text { b } A .39\end{array}$ & $\begin{array}{c}5.60 \pm 0.44 \\
\text { b } A\end{array}$ & $\begin{array}{c}5.64 \pm 0.47 \\
\text { b } \quad \text { A }\end{array}$ & $\begin{array}{c}1.94 \pm 0.41 \\
\text { b } \\
\text { B }\end{array}$ & زيت الشبنت \\
\hline$* *$ & $\begin{array}{c}18.16 \pm 0.50 \\
b\end{array}$ & $\begin{array}{c}5.06 \pm 0.43 \\
\text { b A }\end{array}$ & $\begin{array}{c}5.50 \\
\text { b }\end{array}$ & $\begin{array}{c}5.66 \pm 0.54 \\
\text { b } \quad \text { A }\end{array}$ & $\begin{array}{c}1.94 \pm 0.57 \\
\text { b B }\end{array}$ & زيت الحبة السوداء \\
\hline & $* *$ & $* *$ & $* *$ & $* *$ & $* *$ & تأثيّر المعاملات \\
\hline
\end{tabular}

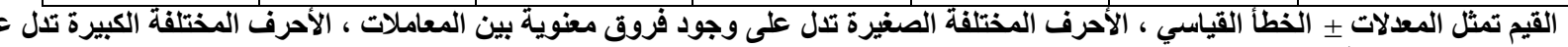

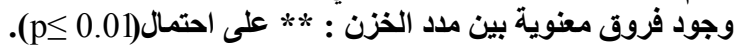

جدول ( 3 ) تأثير المعاملات المختلفة ومدة خزن البيض في الغرفة في معدل قيمة الأس الهيدروجيني .

\begin{tabular}{|c|c|c|c|c|c|c|}
\hline تأثير مدة & \multicolumn{4}{|c|}{ مدة الخزن (أسبوع) } & & \multirow{2}{*}{ المعاملات } \\
\hline الخزن & 8 & 4 & 2 & 1 & طازج & \\
\hline ** & $\begin{array}{c}9.25 \pm 0.32 \\
a \quad A\end{array}$ & $\begin{array}{c}8.86 \pm 0.34 \\
\text { a } \quad \text { B }\end{array}$ & $\begin{array}{c}8.31 \pm 0.41 \\
\text { a } \quad C\end{array}$ & $\begin{array}{c}7.90 \\
\pm 0.36 \mathrm{a} \\
\mathrm{D}\end{array}$ & $\begin{array}{c}7.28 \\
\pm 0.38 \\
\text { E }\end{array}$ & سيطرة \\
\hline ** & $\begin{array}{c}9.02 \pm 0.40 \\
\text { b } A\end{array}$ & $\begin{array}{c}8.17 \pm 0.32 \\
\text { b } \quad \text { B }\end{array}$ & $\begin{array}{c}7.85 \pm 0.40 \\
\text { b } \quad C\end{array}$ & $\begin{array}{c}7.52 \pm 0.31 \\
\text { b } \\
\text { D }\end{array}$ & $\begin{array}{c}7.30 \pm 0.34 \\
E\end{array}$ & زيت الذرة \\
\hline ** & $\begin{array}{c}9.06 \pm 0.38 \\
\text { b } A\end{array}$ & $\begin{array}{c}8.20 \pm 0.36 \\
\text { b } \quad \text { B }\end{array}$ & $\begin{array}{c}7.89 \pm 0.33 \\
\text { b } \quad C\end{array}$ & $\begin{array}{c}7.58 \pm 0.36 \\
\text { b } \\
\text { D }\end{array}$ & $\begin{array}{c}7.31 \pm 0.34 \\
E\end{array}$ & زيت الشبنت \\
\hline ** & $\begin{array}{c}9.03 \pm 0.29 \\
\text { b } A\end{array}$ & $\begin{array}{c}8.18 \pm 0.35 \\
\text { b } B\end{array}$ & $\begin{array}{c}7.88 \pm 0.37 \\
\text { b } \quad C\end{array}$ & $\begin{array}{c}7.55 \pm 0.33 \\
\text { b D }\end{array}$ & $\begin{array}{c}7.30 \pm 0.32 \\
E\end{array}$ & زيت الحبة السوداء \\
\hline & $* *$ & $* *$ & $* *$ & $* *$ & N.S. & تأثيّر المعاملات \\
\hline
\end{tabular}

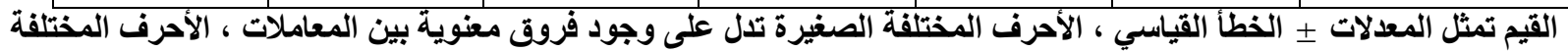

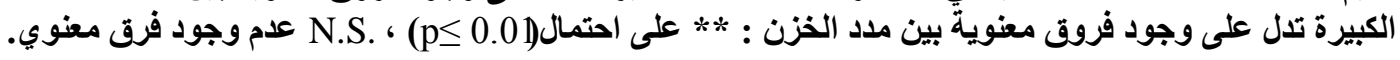


المجلة الطبية البيطرية العراقية 34 ( 2 ): 131 - 141 ، ( 2010 ).

جدول ( 4 ) تأثير المعاملات المختلفة ومدة خزن البيض في الغرفة في قيمة الأحماض الدهنية الحرة (\%) .

\begin{tabular}{|c|c|c|c|c|c|c|}
\hline تأثتير مدة & \multicolumn{4}{|c|}{ مدة الخزن (أسبوع) } & & \multirow{2}{*}{ المعاملات } \\
\hline الخزن & 8 & 4 & 2 & 1 & طازج & \\
\hline ** & $\begin{array}{c}4.79 \pm 0.52 \\
a \quad A\end{array}$ & $\begin{array}{c}3.64 \pm 0.52 \\
\text { a } \quad \text { B }\end{array}$ & $\begin{array}{c}2.19 \pm 0.54 \\
a \mathrm{C}\end{array}$ & $\begin{array}{c}0.91 \pm 0.60 \\
\text { a } \\
\text { D }\end{array}$ & $\begin{array}{c}0.26 \pm 0.61 \\
E\end{array}$ & سيطرة \\
\hline$* *$ & $\begin{array}{c}3.17 \pm 0.55 \\
\text { b } \quad A\end{array}$ & $\begin{array}{c}2.09 \pm 0.50 \\
\text { b } \quad \text { B }\end{array}$ & $\begin{array}{c}1.47 \pm 0.54 \\
\text { b } C\end{array}$ & $\begin{array}{c}0.75 \pm 0.64 \\
\text { b } \\
\text { D }\end{array}$ & $\begin{array}{c}0.31 \\
\pm 0.62 \mathrm{E}\end{array}$ & زيت الذرة \\
\hline$* *$ & $\begin{array}{c}3.11 \pm 0.51 \\
\text { b } A\end{array}$ & $\begin{array}{c}2.00 \pm 0.53 \\
\text { b } \quad \text { B }\end{array}$ & $\begin{array}{c}1.46 \pm 0.54 \\
\text { b } C\end{array}$ & $\begin{array}{c}0.77 \pm 0.44 \\
\text { b } \\
\text { D }\end{array}$ & $\begin{array}{c}0.34 \pm 0.51 \\
E\end{array}$ & زيت الثبنت \\
\hline ** & $\begin{array}{c}3.10 \pm 0.53 \\
\text { b } A\end{array}$ & $\begin{array}{cc}2.01 & \pm 0.52 \\
\text { b } & \text { B }\end{array}$ & $\begin{array}{c}1.46 \pm 0.54 \\
\text { b } C\end{array}$ & $\begin{array}{c}0.74 \pm 0.52 \\
\text { b D }\end{array}$ & $\begin{array}{c}0.32 \pm 0.62 \\
E\end{array}$ & زيت الحبة السوداء \\
\hline & $* *$ & ** & $* *$ & * & N.S. & تأثيّر المعاملات \\
\hline
\end{tabular}

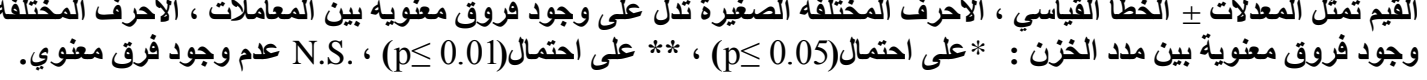

Xدول ( 5 ) تأثير المعاملات المختلفة ومدة خزن البيض في الغرفة في معدل أعداد البكتريا الكلية ( وحدة مكونة للمستعمرة . $10^{3}$

\begin{tabular}{|c|c|c|c|c|c|c|}
\hline تأثير مدة & \multicolumn{4}{|c|}{ مدة الخزن (أسبوع) } & & \multirow{2}{*}{ المعاملات } \\
\hline الخزن & 8 & 4 & 2 & 1 & طازج & \\
\hline ** & $\begin{array}{r}69.85 \\
\pm 6.52 \quad \mathrm{a} \\
\mathrm{A}\end{array}$ & $\begin{array}{c}10.88 \pm 0.32 \\
\text { a } \quad \text { B }\end{array}$ & $\begin{array}{c}0.681 \pm 0.02 \\
\text { a } \quad C\end{array}$ & $\begin{array}{c}0.124 \\
\pm 0.01 \mathrm{a} \\
\mathrm{D}\end{array}$ & $\begin{array}{c}0.00 \\
\pm 0.00 \mathrm{E}\end{array}$ & سيطرة \\
\hline$* *$ & $\begin{array}{c}29.45 \pm 2.65 \\
\text { b A }\end{array}$ & $\begin{array}{c}3.68 \pm 0.10 \\
\text { b } \quad \text { B }\end{array}$ & $\begin{array}{r}0.211 \\
\pm 0.01 \quad \mathrm{~b} \quad \mathrm{C}\end{array}$ & $\begin{array}{c}0.073 \\
\pm 0.01 \mathrm{~b} \\
\mathrm{D}\end{array}$ & $\begin{array}{c}0.00 \\
\pm 0.00 \mathrm{E}\end{array}$ & زيت الذرة \\
\hline$* *$ & $\begin{array}{c}10.43 \pm 2.36 \\
\text { c A }\end{array}$ & $\begin{array}{c}1.24 \pm 0.13 \\
\text { c } \quad \text { B }\end{array}$ & $\begin{array}{c}0.063 \pm 0.02 \\
\text { c } \quad \mathrm{C}\end{array}$ & $\begin{array}{c}0.015 \\
\pm 0.01 \mathrm{c} \\
\mathrm{D}\end{array}$ & $\begin{array}{c}0.00 \\
\pm 0.00 \mathrm{E}\end{array}$ & زيت الثبنت \\
\hline ** & $\begin{array}{c}9.85 \pm 2.08 \\
\text { c } \quad \text { A }\end{array}$ & $\begin{array}{c}1.18 \pm 0.12 \\
\text { c } \quad B\end{array}$ & $\begin{array}{c}0.059 \pm 0.01 \\
\text { c } \quad \mathrm{C}\end{array}$ & $\begin{array}{c}0.012 \\
\pm 0.01 \mathrm{c} \\
\mathrm{D}\end{array}$ & $\begin{array}{r}0.00 \\
\pm 0.00 \quad \mathrm{E}\end{array}$ & زيت الحبة السوداء \\
\hline & $* *$ & $* *$ & $* *$ & $* *$ & N.S. & تأثير المعاملات \\
\hline
\end{tabular}

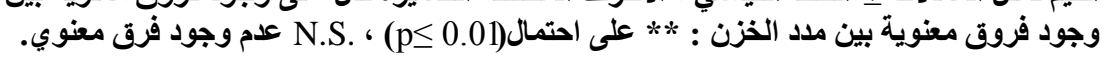


المجلة الطبية البيطرية العراقية 34 ( 2 ): 131 - 141 ، ( 2010 ).

جدول ( 6 ) تأثير المعاملات المختلفة ومدة خزن البيض في الغرفة في معدل أعداد الفطريات (وحدة مكونة للمستعمرة /غم) .

\begin{tabular}{|c|c|c|c|c|c|c|}
\hline تأثير مدة & \multicolumn{4}{|c|}{ مدة الخزن (أسبوع) } & & \multirow{2}{*}{ المعاملات } \\
\hline الخزن & 8 & 4 & 2 & 1 & طازج & \\
\hline$* *$ & $\begin{array}{c}12.96 \pm 4.58 \\
\text { b A }\end{array}$ & $\begin{array}{c}2.35 \pm 0.12 \\
\mathrm{a} \quad \mathrm{B}\end{array}$ & $\begin{array}{c}0.10 \\
\pm 0.00 \text { a C }\end{array}$ & $\begin{array}{r}0.00 \\
\pm 0.00 \mathrm{D}\end{array}$ & $\begin{array}{r}0.00 \\
\pm 0.00 \mathrm{E}\end{array}$ & سيطرة \\
\hline$* *$ & $\begin{array}{c}1.45 \pm 0.75 \\
\text { c } \quad \text { A }\end{array}$ & $\begin{array}{c}0.38 \pm 0.05 \\
\text { b } \quad \text { B }\end{array}$ & $\begin{array}{c}0.00 \\
\pm 0.00 \text { b C }\end{array}$ & $\begin{array}{c}0.00 \\
\pm 0.00 \mathrm{C}\end{array}$ & $\begin{array}{c}0.00 \\
\pm 0.00 \mathrm{C}\end{array}$ & زيت الذرة \\
\hline$* *$ & $\begin{array}{c}0.63 \pm 0.01 \\
\text { с } \quad \mathrm{A}\end{array}$ & $\begin{array}{r}0.00 \\
\pm 0.00 \text { с B }\end{array}$ & $\begin{array}{c}0.00 \\
\pm 0.00 \text { b C }\end{array}$ & $\begin{array}{c}0.00 \\
\pm 0.00 \mathrm{C}\end{array}$ & $\begin{array}{c}0.00 \\
\pm 0.00 \mathrm{C}\end{array}$ & زيت الشبنت \\
\hline$* *$ & $\begin{array}{c}0.50 \pm 0.03 \\
\text { c } \quad A\end{array}$ & $\begin{array}{c}0.00 \\
\pm 0.00 \mathrm{c} \\
\mathrm{B}\end{array}$ & $\begin{array}{c}0.00 \\
\pm 0.00 \text { b C }\end{array}$ & $\begin{array}{c}0.00 \\
\pm 0.00 \mathrm{C}\end{array}$ & $\begin{array}{r}0.00 \\
\pm 0.00 \mathrm{C}\end{array}$ & زيت الحبة السوداء \\
\hline & $* *$ & $* *$ & $*$ & N.S. & N.S. & تأثنير المعاملات \\
\hline
\end{tabular}

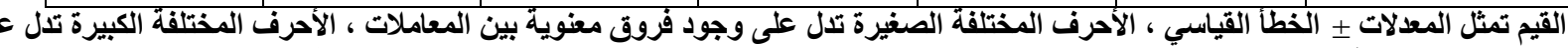

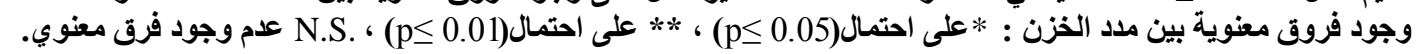

جدول ( 7 ) تأثير المعاملات المختلفة ومدة خزن البيض في الثلاجة في معدل وزن البيضة (غم).

\begin{tabular}{|c|c|c|c|c|c|c|}
\hline تأثنير مدة & \multicolumn{4}{|c|}{ مدة الخزن (أسبوع) } & & \multirow{2}{*}{ المعاملات } \\
\hline الخزن & 8 & 4 & 2 & 1 & طازج & \\
\hline$* *$ & $\begin{array}{c}49.34 \\
\quad \text { b }\end{array}$ & $\begin{array}{c}53.77 \pm 1.17 \\
\text { b } \\
\text { C }\end{array}$ & $\begin{array}{c}58.35 \pm 1.10 \\
\text { b } \\
\text { B }\end{array}$ & $\begin{array}{c}\mathbf{6 0 . 6 9} \\
\pm 1.09 \\
\\
\text { A }\end{array}$ & $\begin{array}{c}61.21 \\
\pm 1.23 \mathrm{~A}\end{array}$ & سيطرة \\
\hline$* *$ & $\begin{array}{c}54.90 \\
\text { a }\end{array}$ & $\begin{array}{c}58.86 \pm 1.20 \\
\text { a } \\
\text { B }\end{array}$ & $\begin{array}{c}60.60 \pm 1.11 \\
\mathrm{a} \\
\mathrm{A}\end{array}$ & $\begin{array}{c}61.11 \\
\pm 1.11 \\
\text { A }\end{array}$ & $\begin{array}{c}61.39 \\
\pm 1.19 \mathrm{~A}\end{array}$ & زيت الذرة \\
\hline$* *$ & $\begin{array}{c}54.99 \pm 1.20 \\
\text { a }\end{array}$ & $\begin{array}{c}58.79 \pm 1.21 \\
\text { a } \\
\text { B }\end{array}$ & $\begin{array}{c}60.74 \pm 1.21 \\
\mathrm{a} \\
\mathrm{A}\end{array}$ & $\begin{array}{c}61.16 \\
\pm 1.19 \\
\text { A }\end{array}$ & $\begin{array}{c}61.47 \\
\pm 1.22 \mathrm{~A}\end{array}$ & زيت الشبنت \\
\hline$* *$ & $\begin{array}{c}54.93 \\
\text { a }\end{array}$ & $\begin{array}{c}58.81 \pm 1.11 \\
\text { a } \\
\text { B }\end{array}$ & $\begin{array}{c}60.69 \pm 1.21 \\
\mathrm{a} \\
\mathrm{A}\end{array}$ & $\begin{array}{c}61.20 \\
\pm 1.17 \\
\text { A }\end{array}$ & $\begin{array}{c}61.46 \\
\pm 1.04 \mathrm{~A}\end{array}$ & زيت الحبة السوداء \\
\hline & $* *$ & $* *$ & $*$ & N.S. & N.S. & تأثيْير المعاملات \\
\hline
\end{tabular}

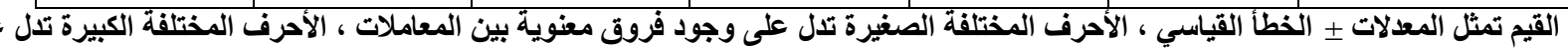

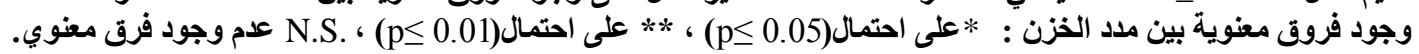


المجلة الطبية البيطرية العراقية 34 ( 2 ): 131 - 141 ، ( 2010 ).

جدول ( 8 ) تأثير المعاملات المختلفة ومدة خزن البيض في الثلاجة في نسبة الفقد في وزن البيضة(\%) .

\begin{tabular}{|c|c|c|c|c|c|c|}
\hline تأثير مدة & \multicolumn{4}{|c|}{ مدة الخزن (أسبوع) } & & \multirow{2}{*}{ المعاملات } \\
\hline الخزن & 1 & 8 & 4 & 2 & 1 & \\
\hline$* *$ & $\begin{array}{c}20.80 \pm 0.48 \\
a\end{array}$ & $\begin{array}{c}8.24 \\
\text { a }\end{array} \quad$ A & $\begin{array}{c}7.85 \pm 0.52 \mathrm{a} \\
\mathrm{B}\end{array}$ & $\begin{array}{c}3.86 \\
\pm 0.59 a \\
C\end{array}$ & $\begin{array}{r}0.85 \\
\pm 0.59 \\
\text { D }\end{array}$ & سيطرة \\
\hline$* *$ & $\begin{array}{c}10.88 \pm 0.51 \\
b\end{array}$ & $\begin{array}{c}6.72 \pm 0.52 \\
\text { b } \quad A\end{array}$ & $\begin{array}{c}2.87 \pm 0.54 b \\
\text { B }\end{array}$ & $\begin{array}{c}0.83 \pm 0.56 \\
b \\
\text { C }\end{array}$ & $\begin{array}{c}0.46 \pm 0.56 \\
C\end{array}$ & زيت الذرة \\
\hline$* *$ & $\begin{array}{c}10.86 \\
b\end{array}$ & $\begin{array}{c}6.46 \pm 0.44 \\
\text { b } A\end{array}$ & $\begin{array}{c}3.21 \pm 0.47 b \\
\text { B }\end{array}$ & $\begin{array}{c}0.69 \pm 0.41 \\
\text { b } \\
\text { C }\end{array}$ & $\begin{array}{c}0.50 \pm 0.41 \\
C\end{array}$ & زيت الثبنت \\
\hline$* *$ & $\begin{array}{c}10.95 \pm 0.50 \\
b\end{array}$ & $\begin{array}{c}6.60 \pm 0.41 \\
b \quad A\end{array}$ & $\begin{array}{c}3.10 \pm 0.54 \mathrm{~b} \\
\text { B }\end{array}$ & $\begin{array}{c}0.83 \pm 0.57 \\
\text { b C }\end{array}$ & $\begin{array}{c}0.42 \pm 0.57 \\
C\end{array}$ & زيت الحبة السوداء \\
\hline & $* *$ & $* *$ & $* *$ & $* *$ & N.S. & تأثثير المعاملات \\
\hline
\end{tabular}

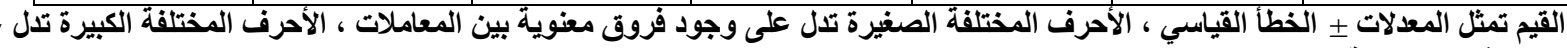

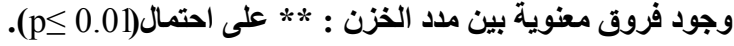

جدول ( 9 ) تأثير المعاملات المختلفة ومدة خزن البيض في الثلاجة في معدل قيمة الأس الهيلروجيني .

\begin{tabular}{|c|c|c|c|c|c|c|}
\hline تأثثير مدة & \multicolumn{4}{|c|}{ مدة الخزن (أسبوع) } & & \multirow{2}{*}{ المعاملات } \\
\hline الخزن & 8 & 4 & 2 & 1 & طازج & \\
\hline$* *$ & $\begin{array}{c}9.07 \pm 0.39 \\
\mathrm{a} \quad \mathrm{A}\end{array}$ & 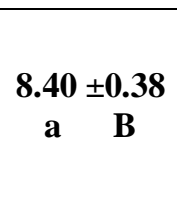 & $\begin{array}{c}7.96 \\
\pm 0.32 \mathrm{a} \\
\mathrm{C}\end{array}$ & $\begin{array}{c}7.57 \\
\pm 0.38 \\
\text { D }\end{array}$ & $\begin{array}{r}7.28 \\
\pm 0.40 \\
\text { E }\end{array}$ & سيطرة \\
\hline$* *$ & $\begin{array}{c}8.28 \\
\text { b }\end{array} \quad$ A & $\begin{array}{c}7.91 \pm 0.36 \\
\text { b } \quad \text { B }\end{array}$ & $\begin{array}{c}7.58 \pm 0.38 \mathrm{~b} \\
\mathrm{C}\end{array}$ & $\begin{array}{c}7.44 \pm 0.36 \\
\text { CD }\end{array}$ & $\begin{array}{c}7.30 \pm 0.38 \\
D\end{array}$ & زيت الذرة \\
\hline$* *$ & $\begin{array}{c}8.32 \pm 0.35 \\
\text { b } A\end{array}$ & $\begin{array}{c}7.93 \pm 0.32 \\
\text { b } \quad B\end{array}$ & $\begin{array}{c}7.57 \pm 0.33 \mathrm{~b} \\
\mathrm{C}\end{array}$ & $\begin{array}{c}7.45 \pm 0.35 \\
\text { CD }\end{array}$ & $\begin{array}{c}7.31 \pm 0.37 \\
\text { D }\end{array}$ & زيت الثبنت \\
\hline$* *$ & $\begin{array}{c}8.31 \pm 0.33 \\
\text { b } \quad A\end{array}$ & $\begin{array}{c}7.90 \pm 0.37 \\
b \quad B\end{array}$ & $\begin{array}{c}7.58 \pm 0.35 \mathrm{~b} \\
\mathrm{C}\end{array}$ & $\begin{array}{c}7.45 \pm 0.30 \\
\mathrm{CD}\end{array}$ & $\begin{array}{c}7.30 \pm 0.37 \\
\mathrm{D}\end{array}$ & زيت الحبة السوداء \\
\hline & $* *$ & $* *$ & $*$ & N.S. & N.S. & تأثّير المعاملات \\
\hline
\end{tabular}

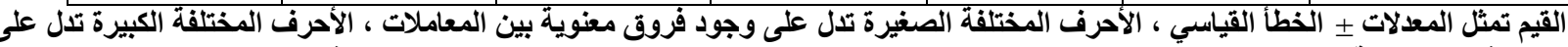
وجود فروق مغنوية بين مدد الخزن : * على احتمال(p 
المجلة الطبية البيطرية العراقية 34 ( 2 ): 131 - 141 ، ( 2010 ).

جدول ( 10 ) تأثير المعاملات المختلفة ومدة خزن البيض في الثلاجة في قيمة الأحماض الدهنية الحرة (\%) .

\begin{tabular}{|c|c|c|c|c|c|c|}
\hline تأثنير مدة & \multicolumn{4}{|c|}{ مدة الخزن (أسبوع) } & & \multirow{2}{*}{ المعاملات } \\
\hline الخزن & 8 & 4 & 2 & 1 & طازج & \\
\hline$* *$ & $\begin{array}{cc}3.41 & \pm 0.54 \\
\text { a } & \text { A }\end{array}$ & $\begin{array}{c}2.23 \pm 0.62 \\
\text { a B }\end{array}$ & $\begin{array}{c}0.95 \pm 0.53 a \\
C\end{array}$ & $\begin{array}{c}0.46 \pm 0.61 \\
\text { D }\end{array}$ & $\begin{array}{c}0.26 \pm 0.53 \\
E\end{array}$ & سيطرة \\
\hline$* *$ & $\begin{array}{c}2.12 \pm 0.58 \\
\text { b } \quad A\end{array}$ & $\begin{array}{c}1.55 \pm 0.60 \\
\text { b } \quad B\end{array}$ & $\begin{array}{c}0.72 \pm 0.56 \text { b } \\
C\end{array}$ & $\begin{array}{c}0.36 \pm 0.58 \\
\text { D }\end{array}$ & $\begin{array}{c}0.31 \\
\pm 0.57 \mathrm{D}\end{array}$ & زيت الذرة \\
\hline$* *$ & $\begin{array}{c}2.13 \pm 0.55 \\
\text { b } \quad A\end{array}$ & $\begin{array}{c}1.56 \pm 0.58 \\
\text { b } \quad \text { B }\end{array}$ & $\begin{array}{c}0.76 \pm 0.50 \text { b } \\
\text { C }\end{array}$ & $\begin{array}{c}0.37 \pm 0.53 \\
\text { D }\end{array}$ & $\begin{array}{c}0.34 \pm 0.55 \\
D\end{array}$ & زيت الشبنت \\
\hline$* *$ & $\begin{array}{c}2.15 \pm 0.55 \\
\text { b } \quad A\end{array}$ & $\begin{array}{c}1.53 \pm 0.57 \\
\text { b } \quad \text { B }\end{array}$ & $\begin{array}{c}0.74 \pm 0.52 \mathrm{~b} \\
\mathrm{C}\end{array}$ & $\begin{array}{c}0.37 \pm 0.57 \\
D\end{array}$ & $\begin{array}{c}0.32 \pm 0.60 \\
D\end{array}$ & زيت الحبة السوداء \\
\hline & $* *$ & $* *$ & $*$ & N.S. & N.S. & تأثنير المعاملات \\
\hline
\end{tabular}

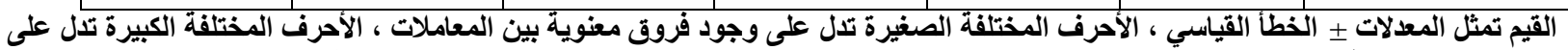

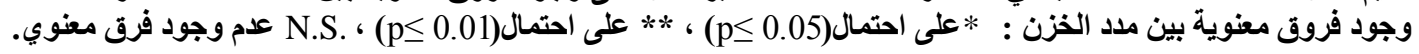

جدول ( 11 ) تأثير المعاملات المختلفة ومدة خزن البيض في الثلاجة في معدل أعداد البكتريا الكلية

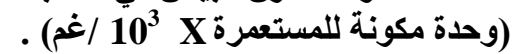

\begin{tabular}{|c|c|c|c|c|c|c|}
\hline تأثثير مدة & \multicolumn{4}{|c|}{ مدة الخزن (أسبو ع) } & & \multirow{2}{*}{ المعاملات } \\
\hline الخزن & 8 & 4 & 2 & 1 & طازج & \\
\hline ** & $\begin{array}{c}9.73 \pm 0.36 \\
\mathrm{a} \quad \mathrm{A}\end{array}$ & $\begin{array}{c}0.590 \pm 0.03 \\
\text { a B }\end{array}$ & $\begin{array}{c}0.117 \pm 0.02 \\
\text { a C }\end{array}$ & $\begin{array}{r}0.012 \\
\pm 0.01 \text { a } D\end{array}$ & $\begin{array}{c}0.00 \\
\pm 0.00 \mathrm{E}\end{array}$ & سيطرة \\
\hline ** & $\begin{array}{c}2.98 \pm 0.14 \\
\text { b } A\end{array}$ & $\begin{array}{r}0.168 \\
\pm 0.02 \quad \text { b } \\
\text { B }\end{array}$ & $\begin{array}{c}0.056 \pm 0.02 \\
\text { b } C\end{array}$ & $\begin{array}{c}0.00 \\
\pm 0.00 \text { b D }\end{array}$ & $\begin{array}{c}0.00 \\
\pm 0.00 D\end{array}$ & زيت الذرة \\
\hline ** & $\begin{array}{c}0.58 \pm 0.17 \\
\text { c } \quad A\end{array}$ & $\begin{array}{c}0.025 \pm 0.03 \\
\text { c } \quad B\end{array}$ & $\begin{array}{c}0.011 \pm 0.02 \\
\text { c } \mathrm{C}\end{array}$ & $\begin{array}{r}0.00 \\
\pm 0.00 \text { b D }\end{array}$ & $\begin{array}{c}0.00 \\
\pm 0.00 D\end{array}$ & زيت الثبنت \\
\hline ** & $\begin{array}{cc}0.51 & \pm 0.11 \\
\text { c } & A\end{array}$ & $\begin{array}{c}0.021 \pm 0.02 \\
\text { c } \quad \text { B }\end{array}$ & $\begin{array}{c}0.011 \\
\text { c }\end{array}$ & $\begin{array}{c}\mathbf{0 . 0 0} \\
\mathbf{\pm 0 . 0 0} \text { b } \\
\text { D }\end{array}$ & $\begin{array}{r}0.00 \\
\pm 0.00 \quad D\end{array}$ & زيت الحبة السوداء \\
\hline & $* *$ & $* *$ & $* *$ & N.S. & N.S. & تأثيّر المعاملات \\
\hline
\end{tabular}

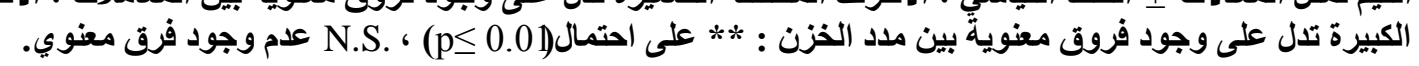


المجلة الطبية البيطرية العراقية 34 ( 2 ): 131 - 141 ، ( 2010 ).

جدول ( 12 ) تأثير المعاملات المختلفة ومدة خزن البيض في الثلاجة في معدل أعداد الفطريات (وحدة مكونة للمستعمرة /غم).

\begin{tabular}{|c|c|c|c|c|c|c|}
\hline تأتثير مدة & \multicolumn{4}{|c|}{ مدة الخزن (أسبوع) } & & \multirow{2}{*}{ المعاملات } \\
\hline الخزن & 8 & 4 & 2 & 1 & طازج & \\
\hline$* *$ & $\begin{array}{c}2.61 \\
\text { a }\end{array}$ & $\begin{array}{c}0.10 \\
\pm 0.00 \text { a B }\end{array}$ & $\begin{array}{c}0.00 \\
\pm 0.00 \\
\mathrm{C}\end{array}$ & $\begin{array}{c}0.00 \\
\pm 0.00 \mathrm{C}\end{array}$ & $\begin{array}{c}0.00 \\
\pm 0.00 \mathrm{C}\end{array}$ & سيطرة \\
\hline$* *$ & $\begin{array}{c}0.74 \\
\text { b }\end{array}$ & $\begin{array}{c}0.00 \\
\pm 0.00 \text { b B }\end{array}$ & $\begin{array}{r}0.00 \\
\pm 0.00 \\
\text { B }\end{array}$ & $\begin{array}{c}0.00 \\
\pm 0.00 \text { B }\end{array}$ & $\begin{array}{c}0.00 \\
\pm 0.00 \mathrm{~B}\end{array}$ & زيت الذرة \\
\hline$* *$ & $\begin{array}{r}0.05 \\
\pm 0.00 \text { c } \quad A\end{array}$ & $\begin{array}{r}0.00 \\
\pm 0.00 \text { b B }\end{array}$ & $\begin{array}{r}0.00 \\
\pm 0.00 \\
\text { B }\end{array}$ & $\begin{array}{c}0.00 \\
\pm 0.00 \text { B }\end{array}$ & $\begin{array}{c}0.00 \\
\pm 0.00 \mathrm{~B}\end{array}$ & زيت الثبنت \\
\hline$* *$ & $\begin{array}{c}0.02 \\
\pm 0.00 \mathrm{c} \\
\mathrm{A}\end{array}$ & $\begin{array}{c}0.00 \\
\pm 0.00 \text { b B }\end{array}$ & $\begin{array}{r}0.00 \\
\pm 0.00 \\
\text { B }\end{array}$ & $\begin{array}{c}0.00 \\
\pm 0.00 \text { B }\end{array}$ & $\begin{array}{c}0.00 \\
\pm 0.00 \text { B }\end{array}$ & زيت الحبة السوداء \\
\hline & $* *$ & * & N.S. & N.S. & N.S. & تأثّير المعاملات \\
\hline
\end{tabular}

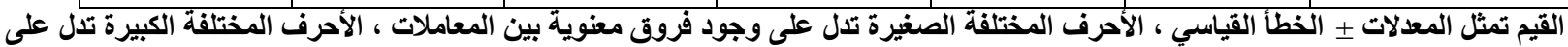

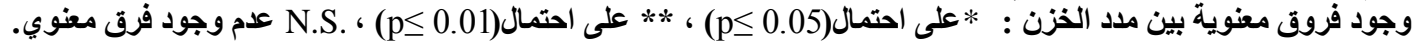

إن أعداد البكتريا و الفطريات في المحتويات الداخلية للبيض الطاز ج و المخزون لمعاملات الزيوت الطيات الطبية كان منخفضا

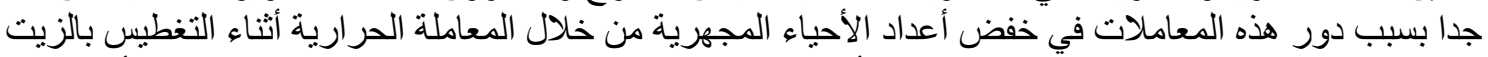

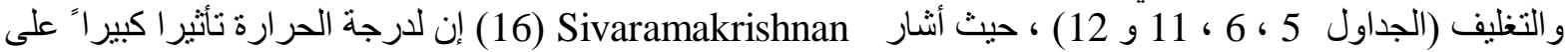

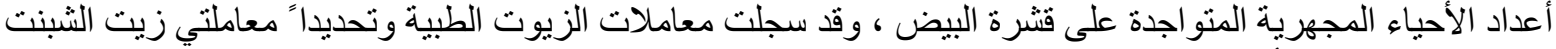

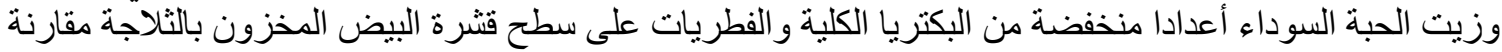

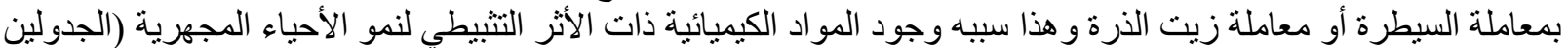

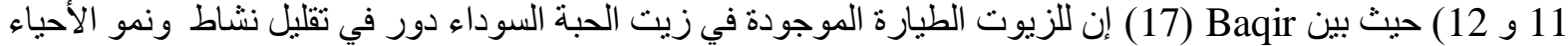

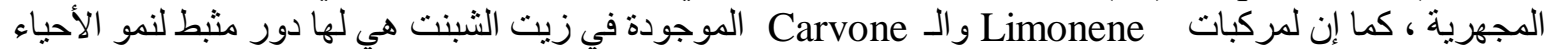

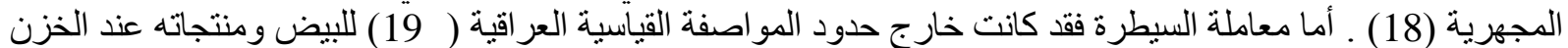

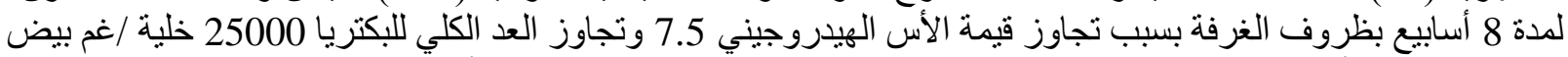

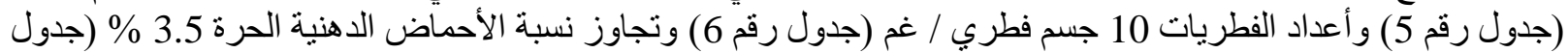

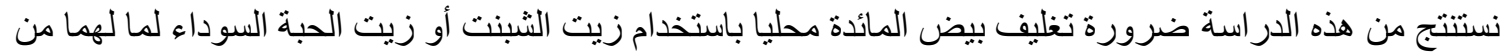

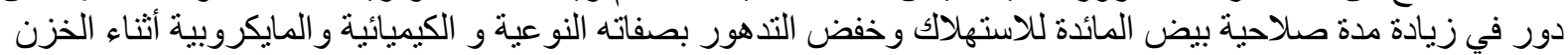
وخاصة خلال فصل الصيف.

\section{المصادر}

1. Stadelman WJ and Cotterill OJ (1995) . Egg Science and Technology. $4^{\text {th }}$ ed. Food Products Press. An Imprint of the Haworth Press. INC. New York. London.

2. USDA United State Department of Agriculture (2000) . Egg Grading Manual . MPHotline.fsis@usda.gov 
3. Hank CR Kunkel ME Dawson PL Acton JC and Wardlaw F B (2001). The effect of shell egg pasteurization on the protein quality of albumen. Poultry Sci.80 : 821-825.

4. ألعبيدي ، فارس عبد علي وصبري جثير وشهرز اد محمد جعفر ألثديدي وسمير عبد الله الازرقي (2007 ) ـ

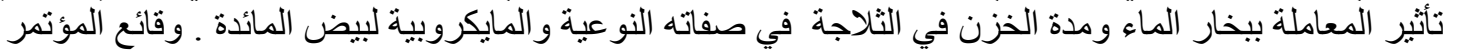

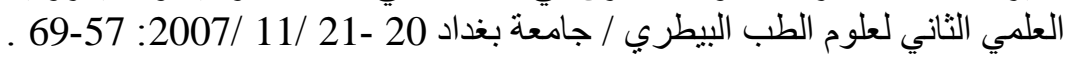

5. Wilburn D (2006) . Pasteurization help to kill bacteria in egg recipe dishes . Poultry Today, 1-2. dwilburn@uga.edu

6. USDA, United State Department of Agriculture (2007) . Shell egg from farm to table . MPHotline.fsis@usda.gov

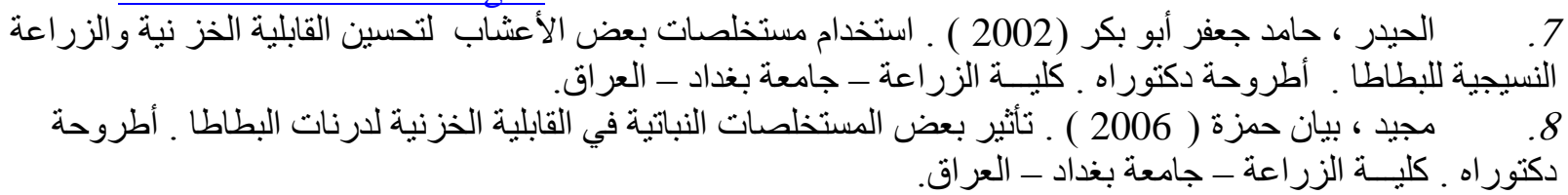

9. Egan H Kirk RS and Sawyer R (1981). Pearsons Chemical Analysis of Food . Churchill, Livingston.

10. Yousef AE and Carlstrom C (2003) . Food Microbiology . A laboratory manual. A John Wiley and Sons INC Publication. Ohio State University . USA.

11. Duncan DB (1955) . Multiple range and multiple test . Biometrics . $11: 1-42$.

12. SAS (2001) .SAS / TAT Users Guide, SAS Institute Inc Cary NC USA.

13. Scott TA and Silversides FG (2000) . The effect of storage and strain of hen on egg quality . Poultry Sci. $79: 1725-1729$.

14. Silversides FG and Scott TA (2001) . Effect of storage and layer age on quality of eggs from two lines of hens. Poultry Sci. $80: 1240-1245$.

15. Jones DR (2007) . Egg functionality and quality during long term storage. Internat $\mathbf{J}$ Poul Sci. $3: 157-162$.

16. Sivaramakrishnan SR (2007) . Microwave pasteurization of shell eggs. MSC thesis submitted to the McGill University Quebec Canada .

17. Baqir AW Al-Ani AH and Al-Kaisey MT (2002). Inhibitory action of Iraq Nigella sativa seed extracts against some pathogenic microorganism . Special symposium for Black seed researchers $15^{\text {th }}$ May.College of Pharmacology.

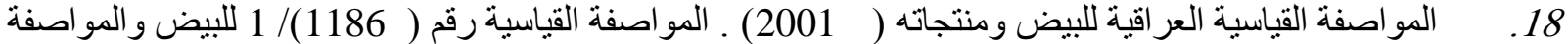

القياسية رقم (1186) /2 لبياض البيض وصفار البيض ، الجهاز المركزي للتقييس والسيطرة النوعية . جمهورية العراق ـ 